\title{
THE HIGH-REDSHIFT CLUSTERS OCCUPIED BY BENT RADIO AGN (COBRA) SURVEY: THE Spitzer CATALOG
}

\author{
R. Paterno-Mahler,${ }^{1,2}$ E. L. Blanton,${ }^{1}$ M. Brodwin, ${ }^{3}$ M. L. N. Ashby,${ }^{4}$ E. Golden-Marx,${ }^{1}$ B. Decker,${ }^{3}$ \\ J. D. WING, ${ }^{4}$ AND G. ANAND ${ }^{1}$
}

\footnotetext{
${ }^{1}$ Astronomy Department and Institute for Astrophysical Research, Boston University, 725 Commonwealth Ave, Boston, MA 02215, USA

${ }^{2}$ Department of Astronomy, University of Michigan, 1085 South University Drive, Ann Arbor, MI 48109, USA

${ }^{3}$ Department of Physics and Astronomy, University of Missouri-Kansas City, 5110 Rockhill Road, Kansas City, MO 64110, USA

${ }^{4}$ Harvard-Smithsonian Center for Astrophysics, 60 Garden Street, Cambridge, MA 02138, USA
}

त

ก

We present 190 galaxy cluster candidates (most at high redshift) based on galaxy overdensity measurements in the Spitzer/IRAC imaging of the fields surrounding 646 bent, double-lobed radio sources drawn from the Clusters Occupied by Bent Radio AGN (COBRA) Survey. The COBRA sources were chosen as objects in the VLA FIRST survey that lack optical counterparts in the Sloan Digital Sky Survey (SDSS) to a limit of $m_{r}=22$, making them likely to lie at high redshift. This is confirmed by our observations: the redshift distribution of COBRA sources with estimated redshifts peaks near $z=1$, and extends out to $z \approx 3$. Cluster candidates were identified by comparing our target fields to a background field and searching for statistically significant $(\geq 2 \sigma)$ excesses in the galaxy number counts surrounding the radio sources; 190 fields satisfy the $\geq 2 \sigma$ limit. We find that 530 fields $(82.0 \%)$ have a net positive excess of galaxies surrounding the radio source. Many of the fields with positive excesses but below the $2 \sigma$ cutoff are likely to be galaxy groups. Forty-one COBRA sources are quasars with known spectroscopic redshifts, which may be tracers of some of the most distant clusters known.

Keywords: galaxies:clusters:general - galaxies:high-redshift - galaxies:evolution - infrared:galaxies radio continuum:galaxies 


\section{INTRODUCTION}

Galaxy clusters are the largest gravitationally bound structures in the universe. They are composed of galaxies, hot X-ray emitting gas, and dark matter. Clusters can be used to investigate many properties of our Universe, including the large-scale structure and dark matter distribution, galaxy formation and evolution, and cosmological parameters. To explore these properties, it is useful to have a sample of clusters covering a large range of redshifts with a well-characterized selection technique. Clusters are commonly detected via a variety of methods, each of which has its strengths and weaknesses. These include finding galaxy overdensities in optical and infrared surveys (the earliest of which were done by Abell (1958) and Zwicky et al. (1961) in the optical), detecting extended emission in Xray surveys (e.g., Vikhlinin et al. 2009), and measuring Sunyaev-Zel'dovich decrements in radio surveys (e.g., Vanderlinde et al. 2010; Marriage et al. 2011; Hasselfield et al. 2013; Bleem et al. 2015). Thousands of clusters have spectroscopic redshifts measured at $z<0.25$, and an increasing number of large surveys are searching for clusters at high redshift (see many of the references mentioned in this section). However, the number of spectroscopically confirmed clusters with redshifts $z>1.0$ is still relatively small.

Clusters selected at optical wavelengths are identified on the basis of two-dimensional galaxy overdensities, but projection effects can cause overcounting. In addition to galaxy overdensities, at optical wavelengths the red sequence can be used to identify clusters (Gladders \& Yee 2000). From the color of the red sequence, an approximate redshift of the cluster can be calculated. At higher redshifts, however, it becomes difficult to identify cluster members, as they become too faint to detect. Additionally, the peak of the galaxy spectral energy distribution shifts to the infrared, causing galaxies to drop out of optical surveys. It is also the case that at high redshift, clusters may not have prominent red sequences yet, as many galaxies may still be forming stars (McGee et al. 2009; Brodwin et al. 2013; Hennig et al. 2016).

Infrared telescopes such as the Spitzer Space Telescope (Werner et al. 2004) can be used to find new clusters with $z>1$ (e.g., Stanford et al. 2005; Brodwin et al. 2006; Muzzin et al. 2009), as the peak emission from galaxies gets redshifted into the infrared. Infrared surveys select galaxies via their stellar mass. Owing to a beneficial K-correction, galaxies observed in the $4.5 \mu \mathrm{m}$ band with Spitzer do not fade with redshift out to at least $z \sim 1.4$ (e.g., Mancone et al. 2010). This method is also independent of the star formation rate of the cluster galaxies (unless a secondary cut is applied, such as the optical/IR color cut in Muzzin et al. (2008)), and thus will also find clusters whose red sequences are just starting to build up, or that do not have a red sequence, which is the case as one moves to higher redshift. Spitzer has allowed several large $z>1$ cluster surveys (e.g., Eisenhardt et al. 2008; Muzzin et al. 2008; Papovich 2008; Stanford et al. 2012; Zeimann et al. 2012; Rettura et al. 2014). While Spitzer enables very high-redshift searches over relatively small areas, the Wide-field Infrared Survey Explorer (WISE) is enabling an all-sky search for massive clusters at $z \sim 1$ (e.g., Stanford et al. 2014; Brodwin et al. 2015; Gonzalez et al. 2015); however, the spatial resolution of WISE is much poorer than that of Spitzer.

In the X-ray regime, galaxy clusters are the most common bright, extended extragalactic sources detected in surveys. Clusters are identified through detection of the $\mathrm{X}$-ray emitting gas in the intracluster medium (ICM). The ICM is compressed and heated by the gravitational potential of the cluster and hence is a very clean cluster observable. These extended sources are rare and so suffer much less from projection effects. While X-ray samples are complete at low redshifts (Reiprich \& Böhringer 2002), X-ray observations of clusters require long exposure times, especially at high redshift, since the surface brightness declines as $(1+z)^{4}$. Thus, flux-limited surveys are biased towards the most luminous (massive) clusters (however, see Churazov et al. 2015, who argue that the higher temperature and density of highredshift clusters compensates for the surface brightness dimming). Additionally, they are also biased towards relaxed clusters, as those clusters that are not relaxed tend to have a less centrally peaked surface brightness profile.

Both optical and X-ray surveys become more costly at high redshifts; however, the Sunyaev-Zel'dovich (SZ, Sunyaev \& Zeldovich 1970) effect is nearly independent of cluster redshift and thus can probe the high-redshift regime. The $\mathrm{SZ}$ effect is the distortion of the cosmic microwave background (CMB) spectrum as CMB photons are inverse Compton scattered as they pass through the ICM. By looking for these distortions, new clusters can be found (Bleem et al. 2015; Hasselfield et al. 2013). The strength of the signal is proportional to the mass of the cluster, so lower-mass clusters may not be detected. Additionally, bright radio point sources such as active galactic nuclei (AGN) can weaken the SZ signature for lower-mass clusters at high redshifts (Lin \& Mohr 2007). Galametz et al. (2009) and Martini et al. (2013) showed that the AGN fraction of clusters increases with redshift, which would make this effect more common at higher redshift.

Previous studies have found that radio sources, particularly bent, double-lobed radio sources, are frequently associated with galaxy clusters (Blanton et al. 2000, 2001, 2003; Giacintucci \& Venturi 2009; Wing \& Blanton 2011; Lakhchaura et al. 2011; Norris 2011; Dehghan et al. 2014). The radio lobes of these AGN are most likely bent because of the ram pressure that occurs due to the relative motion of the AGN host galaxy and the ICM, which makes them good tracers for finding galaxy 
clusters. There are a few possible reasons for this relative motion. First, it is possible that the lobes are bent by the ICM as the galaxy moves through it with a large peculiar velocity. A second explanation is that the ICM is disrupted by a recent large-scale cluster-cluster merger (Burns 1990). In this scenario, a galaxy with a low peculiar velocity encounters the large-scale bulk flow of the ICM, which is enough to bend the lobes. Lastly, these bent, double-lobed radio sources can be found in clusters that are relatively relaxed on large scales, such as Abell 2029 (Clarke et al. 2004; Paterno-Mahler et al. 2013). In such clusters, the sloshing of the ICM (which is related to the merger history of the cluster, Ascasibar \& Markevitch 2006) may cause the bending of the radio lobes. In addition to bent, double-lobed sources, powerful radio sources at high redshift that don't exhibit bending are also frequently associated with the cluster environment (Venemans et al. 2007; Wylezalek et al. 2013, 2014). It may be that the protocluster environment promotes high accretion rates at the scale necessary to generate radio-loud AGN (Hatch et al. 2014). It is also possible that these objects are associated with protoclusters because they are among the most massive galaxies in the universe, and thus inhabit regions of the greatest overdensities, where cluster formation occurs (Miley \& De Breuck 2008).

Because of their frequent association with clusters and groups, and the ease of detecting them in short exposures in large radio surveys, bent, doubled-lobed radio sources make ideal tracers for finding high-redshift clusters. Unlike many other cluster-finding methods, whose mass limit depends on the survey depth in the cluster mass observable, bent, double-lobed radio sources can be found in associations over a wide mass range, from lower-mass groups to the most massive clusters. They are also found in a wide variety of environments, from clusters that are actively undergoing mergers (e.g., Douglass et al. 2011) to clusters that are highly relaxed on large scales (e.g., Paterno-Mahler et al. 2013). Additionally, by the nature of their selection, the clusters in this sample will all contain radio-bright AGN, making them sources in which we can study AGN feedback. Similar cluster searches have been done using powerful radio sources without regard to their morphologies (Galametz et al. 2012; Wylezalek et al. 2013, 2014).

Here we present the initial results of the Clusters Occupied by Bent Radio AGN (COBRA) survey. In $\S 2$ we present the sample. In $\S 3$, we present our Spitzer data, and in $\S 4$, we discuss the number of candidate clusters found. For cluster candidates observed in both the $3.6 \mu \mathrm{m}$ and $4.5 \mu \mathrm{m}$ bands we were able to make initial photometric redshift estimates, which we present in $\S 5$. In $\S 6$ we present representative examples of our cluster candidates, and in $\S 7$ we present our conclusions. Throughout, we assume a cosmology with $H_{0}=70 \mathrm{~km} \mathrm{~s}^{-1} \mathrm{Mpc}^{-1}, \Omega_{\Lambda}=0.7$, and $\Omega_{M}=0.3$. All cosmological distances were calculated using the on- line Cosmological Calculator (Wright 2006). Magnitudes are given using the $\mathrm{AB}$ system and were calculated using SExtractor's automatic photometry routine ${ }^{1}$ (MAG_AUTO). The full COBRA catalog is available as an online supplement to this paper.

\section{THE HIGH-REDSHIFT COBRA SAMPLE}

Using the Faint Images of the Radio Sky at Twenty Centimeters (FIRST; Becker et al. 1995) survey, Wing \& Blanton (2011) created four samples of radio sources and examined their optical environments. The FIRST survey covers $\sim 25 \%$ of the sky and is mostly contained in the northern Galactic cap. It has a flux density threshold of $1 \mathrm{mJy}$, systematic astrometric errors $<0.05^{\prime \prime}$, spatial resolution of $5^{\prime \prime}$, and total positional errors on the order of $\sim 1^{\prime \prime}$. Each sample created by Wing \& Blanton (2011) consisted of a unique selection criterion: visualbent, auto-bent, straight, and single-component. These samples were then cross-correlated with the Sloan Digital Sky Survey (SDSS) to find optical matches to a limit of $m_{r}=22$ to the presumed radio cores. Sources associated with the bent radio sources without optical matches are likely to be distant and form the high-redshift COBRA sample.

The visual-bent sample was compiled by visually examining a sample of $\sim 32,000$ multiple-component radio sources from the 1997 April release of the FIRST catalog (Blanton 2000). Visual-bent sample sources were defined as those that have two or more radio components and a bent morphology. From this, 384 sources were identified as bent, double-lobed sources. Of those, 272 had unique matches in the SDSS. The 272 objects are included in the low-redshift COBRA sample (Wing \& Blanton 2011); however in this work we consider only the 112 objects with $m_{r}$ fainter than 22 , as they are likely to lie at high redshift.

The auto-bent sample was created by using a pattern recognition program (Proctor 2006) to identify bent, double-lobed sources over the entire FIRST catalog (as of 2003 April). The sources in the auto-bent sample all contain three radio components (nominally a core and two lobes). The central component of these automatically detected sources is defined as the component opposite the longest side when making a triangle of the three components. This sample contains 1546 sources of which 599 have unique SDSS matches. There are 94 sources in the auto-bent sample that overlap with the visual-bent sample. For more detail, see Wing \& Blanton (2011). As with the visual-bent sample, we only consider sources without SDSS matches as defined in Wing \& Blanton (2011). This set of sources was further trimmed to arrive at the sample of 653 targets (see below). In both the visual-bent and auto-bent samples the

\footnotetext{
${ }^{1}$ SExtractor User's Manual, v2.13
} 
distance between any two components was limited to be no more than $60^{\prime \prime}$.

In Wing \& Blanton (2011), the straight sample consists of all of the straight, three-component sources in the FIRST survey region. The straight sources have an opening angle greater than $160^{\circ}$, as compared to the bent sources, which have an opening angle less than $160^{\circ}$.

The single-component sample acts as a control. If bent, double-lobed radio sources are preferentially associated with galaxy clusters, then the single-component sample should be associated with clusters at a lower rate than the bent sample. The 782 sources in the single-component sample were randomly selected from the FIRST catalog and have no other radio source within $60^{\prime \prime}$. They also have matches in the SDSS. We do not consider either the straight sample or the singlecomponent sample in this work.

Wing \& Blanton (2011) cross-correlated their samples of radio sources with SDSS to examine their optical environments. They found that the visual-bent sample was associated with clusters or groups with 20 or more member galaxies within a $1 \mathrm{Mpc}$ radius of the radio source with an absolute $r$-magnitude brighter than $M_{r}=-19$ $78 \%$ of the time. The association rate drops to $59 \%$ for the auto-bent sample, $43 \%$ for the straight sample, and $29 \%$ for the single-component sample. If we only include richer systems with 40 or more member galaxies the association rates are $62 \%$ for the visual-bent sample, $41 \%$ for the auto-bent sample, $24 \%$ for the straight sample, and $10 \%$ for the single-component sample.

From the original Wing \& Blanton (2011) sample, PI Blanton visually examined overlays of FIRST radio contours over SDSS images and eliminated the most obvious very low-redshift sources and sources that were not likely to be true bent, double-lobed radio sources. This selection process identified 653 bent, double-lobed radio sources (including sources from the visual- and autobent samples) that did not have obvious detected optical galaxy hosts in the SDSS in the r-band to the limit of $m_{r}=22$ or were detected as blue quasar-like objects and have a known spectroscopic redshift $z>0.7$. For examples of the objects in the sample, see Figure 8 in $\S 6$. Most of the bent, double-lobed radio sources that had matches in SDSS consistent with elliptical galaxies found by Wing \& Blanton (2011) have redshift $z<0.7$. Since the detection limit of SDSS used in Wing \& Blanton (2011) is $m_{r}=22$, if any of the radio sources in the sample of 653 SDSS non-detections have counterparts that are consistent with elliptical galaxies, the elliptical galaxy will be fainter than $m_{r}=22$ in the SDSS and/or is associated with a different component of the radio emission than was assumed in Wing \& Blanton (2011). Thus the radio sources without known optical identifications are most likely at high redshift. There are some cases where the host galaxy was identified offset from the presumed radio core using Spitzer data and some of these hosts have $m_{r}<22$ as discussed in $\S 5$. The original cross-correlation in Wing \& Blanton (2011) using SDSS Data Release 7 (Abazajian et al. 2009) yielded 32 blue, quasar-like objects with spectroscopic redshifts in the SDSS and 621 candidates without an optical host based on the earlier cross-correlation. Objects designated as quasars were designated as such because of their blue color and point-source appearance. Of these 653 sources, 646 were successfully observed in the infrared in our Spitzer snapshot program (§3.1): 511 were observed only at $3.6 \mu \mathrm{m}$, and 135 (including the 32 quasars) were observed at both $3.6 \mu \mathrm{m}$ and $4.5 \mu \mathrm{m}$. A detailed analysis using visual identification of the radio host object and correlation with SDSS Data Release 12 yielded 9 more sources spectroscopically identified as quasars (however, these objects were only observed at $3.6 \mu \mathrm{m}$ ), for a total of 41 quasars and 605 radio AGN not identified as quasars. Together, these 646 sources make up the high-redshift portion of the COBRA survey; the sources with SDSS matches analyzed in Wing \& Blanton (2011) make up the low-redshift portion.

\section{DATA REDUCTION}

\subsection{Spitzer Observations}

The Infrared Array Camera (IRAC; Fazio et al. 2004) observations of the COBRA sources were carried out as a Snapshot Program during Cycle 8 (PID 80161, PI Blanton) using standard observing parameters. Each of the fields targeted by our program was observed with multiple dithered full-array exposures; the number of exposures was adjusted for each source to achieve comparable on-source $\mathrm{S} / \mathrm{N}$ ratios under different background conditions. A medium dither throw was used. All 646 sources were imaged at $3.6 \mu \mathrm{m}$. Two different exposure times were chosen for the sources observed at $3.6 \mu \mathrm{m}$ only: $5 \times 30 \mathrm{~s}$ for sources estimated to have low background and $7 \times 30 \mathrm{~s}$ for sources estimated to have a higher background. We also observed 135 sources at $4.5 \mu \mathrm{m}$. The sources observed at both $3.6 \mu \mathrm{m}$ and $4.5 \mu \mathrm{m}$ were observed for $4 \times 100 \mathrm{~s}$ in both bands. The exposure times for the sources observed at $3.6 \mu \mathrm{m}$ only were calculated with the goal of reaching a limiting $3.6 \mu \mathrm{m}$ flux density of $5 \mu \mathrm{Jy}\left(\mathrm{M}^{*}+1.5\right)$ with a $\mathrm{SNR}=5$. The targeted limiting flux density for the fields observed for $4 \times 100 \mathrm{~s}$ is $3.6 \mu \mathrm{Jy}$. See $\S 4.1$ for a description of our adopted survey depth. These 135 sources include all the sources originally identified as quasars, sources for which we had existing complementary data (in either the optical or NIR), and sources that were deemed especially good candidates because of the appearance of their radio morphologies (particularly clear cases of bent, symmetric, double-lobed sources). In total, 646 distinct COBRA targets were observed between 2011 July and 2013 March.

The IRAC imaging was reduced using standard techniques, using as a starting point the corrected basic calibrated data (cBCD). The cBCD frames were object- 
masked and median-stacked on a per-AOR (Astronomical Observing Request) basis; the resulting stacked images were then visually inspected and subtracted from individual cBCDs within each AOR. This was done to eliminate long-term residual images arising from prior observations of bright sources by the $3.6 \mu \mathrm{m}$ and $4.5 \mu \mathrm{m}$ arrays. Subtracting the median stacks also minimized gradients in the celestial backgrounds around each source. After these preliminaries, the data for each target were mosaicked into spatially registered mosaics using IRACproc (Schuster et al. 2006). IRACproc was configured to automatically flag and reject cosmic ray hits based on pipeline-generated masks together with a sigma-clipping algorithm for spatially coincident pixels. The cBCD frames were resampled to $0 . \prime 6$ pixel $^{-1}$ during mosaicking, so each pixel in the final mosaic subtends one-fourth the solid angle of the native IRAC pixels. The resulting mosaics and coverage maps were subsequently used as the basis for the photometric measurements described below.

\subsection{Source Extraction}

We used SExtractor (Bertin \& Arnouts 1996) in single-image mode on the $3.6 \mu \mathrm{m}$ cutout frames for all observations. We used frames trimmed to 4.5 on a side. We used many of the same parameters as outlined in Lacy et al. (2005), with a few changes. We did apply a filter, using the tophat_2.0_3x3.conv file that is included with SExtractor. The mosaics have a pixel scale of $00^{\prime \prime} 6$ pixel $^{-1}$, so the tophat filter has a FWHM of 1 !' 2 , which is smaller than the IRAC FWHM $\left(1{ }^{\prime \prime} 95\right)$. This smooths the image before detection, making it easier to detect faint objects. We also used a DEBLEND_MINCONT value of 0.0001, a BACK_SIZE value of 25 pixels, and applied the gain appropriate for our observations, based on the number of exposures and exposure time of each field. The parameter DEBLEND_MINCONT is the minimum contrast used when deblending pixels into different objects, and BACK_SIZE is the size (in pixels) of the area used to estimate the background. We ran SExtractor on the cutouts of our Spitzer images and created catalogs of the positions of each detected source, along with the fluxes and magnitudes of each detected source.

When $4.5 \mu \mathrm{m}$ observations were available, we ran SExtractor in dual-image mode, using the $3.6 \mu \mathrm{m}$ image as the reference. We used the same parameters described above. These catalogs were used for estimating redshifts (see $\S 5$ ).

\section{CLUSTER CANDIDATES}

We measured galaxy overdensities in the $3.6 \mu \mathrm{m}$ observations of the COBRA fields by counting sources within $1^{\prime}$ and $2^{\prime}$ of our radio positions ${ }^{2}$, and compar-

${ }^{2}$ At a redshift of $z=1.0,1^{\prime}=480 \mathrm{kpc}$. ing the results to mean galaxy counts at our search depth (see $\$ 4.1$ ) in the deeper IRAC mosaics of the Spitzer UKIDSS Ultra Deep Survey (SpUDS, PI: J. Dunlop). COBRA fields with galaxy number counts greater than $2 \sigma$ (formally, $1.95 \sigma$ ) in excess of the background mean densities were identified as cluster candidates. In compact, rich clusters, the core radius is $R_{c} \simeq(0.1-0.3) h^{-1} \mathrm{Mpc}$ (Bahcall 1975; Dressler 1978; Sarazin 1988). This corresponds to an angular extent of $\sim 1^{\prime}$ at $z=1.0$, which is the expected peak of the redshift distribution of our sample. We extend the search to $2^{\prime}$ to account for the possibility that some of the AGN in our sample reside at the cluster outskirts, rather than at the center of the cluster.

\subsection{Mean Background Counts}

To determine the mean background counts, we used the SpUDS field. The SpUDS field covers the same approximately one square-degree patch of sky that the UKIDSS Ultra Deep Survey does, and consists of deep IRAC and $24 \mu \mathrm{m}$ MIPS observations. We performed the same source extraction as described in $\S 3.2$ on the final $3.6 \mu \mathrm{m}$ SpUDS mosaics to ensure consistency in our analyses.

The SpUDS field is much deeper than our COBRA fields, so we used it to determine our magnitude limit. To do this, we compared the number of detected sources per square arcminute per $\mu \mathrm{Jy}$ bin for each of our Spitzer observation times and the SpUDS field, as shown in Figure 1, following the methodology of Wylezalek et al. (2013), and described below. Figure 1 shows that at $9.6 \mu \mathrm{Jy}$, our $5 \times 30 \mathrm{~s}$ COBRA fields, which are the shallowest in our survey, have $95 \%$ of the number counts of the SpUDS field. This corresponds to $m_{3.6}=21.4$, which we adopt as our limiting magnitude. At this magnitude our fields with $7 \times 30 \mathrm{~s}$ and $4 \times 100 \mathrm{~s}$ exposure times have at least $95 \%$ of the sources seen in the SpUDS field.

To measure the mean background surface count density, we counted up all of the sources in the SExtractor catalog within the 612 one arcminute regions that we placed on the SpUDS field, as shown in Figure 2, to $m_{3.6}=21.4$. Figure 17 in Ashby et al. (2015) shows that at the depth of the COBRA survey, the number count for Milky Way stars is approximately $0.3 \mathrm{ob}-$ jects $\mathrm{mag}^{-1} \operatorname{arcmin}^{-2}$, which is a factor of approximately 100 below the number count we measure for the galaxies in the SpUDS field. Thus no attempt was made to correct for the small contamination due to foreground stars. We also avoided areas of the SpUDS field with bright foreground stars, as seen in Figure 2. Because the SpUDS field is quite large, it is likely to contain groups and clusters. To avoid having such structures affect our measurement of the mean density of the background field, we fitted a Gaussian to the lower half of the distribution of the SpUDS fields galaxy surface area densities (the dashed red histogram to the left of 


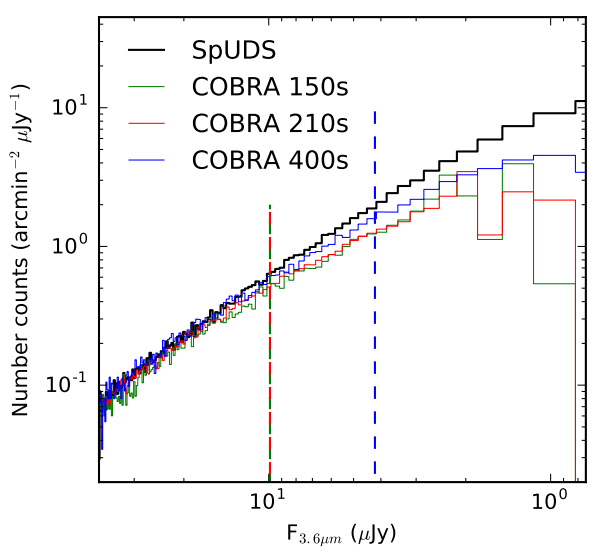

Figure 1. Number counts per square arcminute per $\mu \mathrm{Jy}$ for the different COBRA exposure times (green: $5 \times 30 \mathrm{~s}$, red: $7 \times 30 \mathrm{~s}$, blue: $4 \times 100 \mathrm{~s}$ ) and SpUDS (solid black) fields. The vertical dashed lines show the flux at which COBRA number counts are $95 \%$ those of the SpUDS survey.

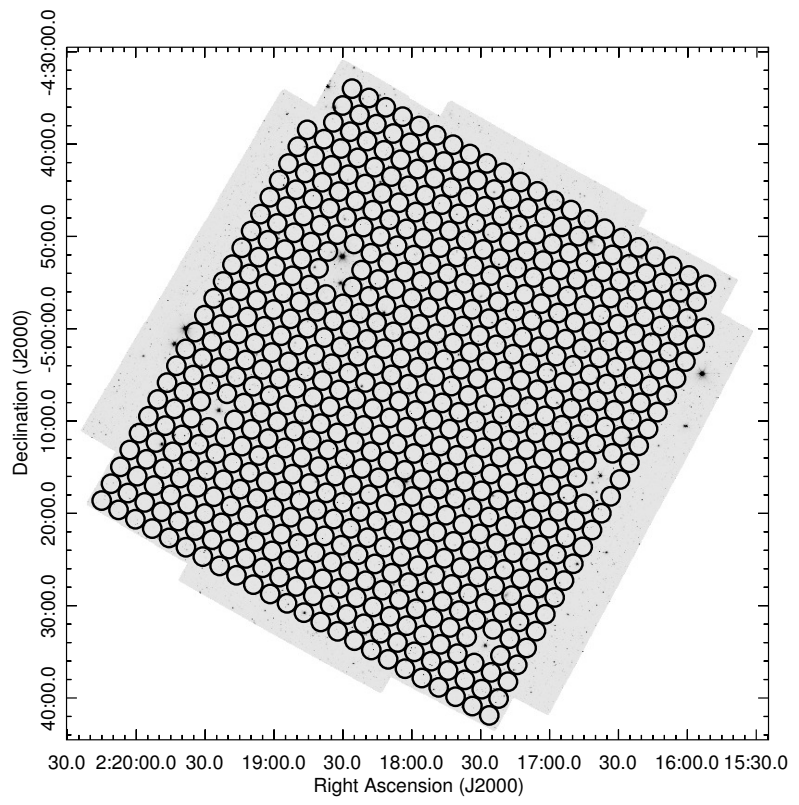

Figure 2. The SpUDS field with 612 regions of $1^{\prime}$ radius overlaid. Areas where bright foreground objects could possibly contaminate the source list were not included.

the vertical line in Figure 3; the solid red curve shows the Gaussian fit), following the method described in Galametz et al. (2012). We find that the average background surface density is 8.5 sources $\operatorname{arcmin}^{-2}$ (26.7 sources per one arcminute radius region) with a standard deviation $\left(\sigma_{S P U D S}\right)$ of 2.0 sources $\operatorname{arcmin}^{-2}(6.3$ sources per one arcminute radius region). We performed a similar analysis on the SpUDS fields with regions two arcminutes in radius. Using 152 two arcminute regions we find a mean background surface density of 8.4 sources $\operatorname{arcmin}^{-2}$ (105.6 sources per two arcminute radius region) and a standard deviation $\left(\sigma_{S p U D S}\right)$ of 1.2 sources $\operatorname{arcmin}^{-2}$ (15.1 sources per two arcminute radius region).

\subsection{Identifying Cluster Candidates}

Candidate clusters were identified on the basis of excess galaxy counts measured in the IRAC mosaics. Specifically, we counted IRAC-detected sources within radii of both $1^{\prime}$ and $2^{\prime}$ of each COBRA radio source and compared these counts to the background counts from SpUDS. As discussed in Wing \& Blanton (2013), the radio sources in the low-redshift COBRA sample are located at both the center and the outskirts of the clusters, which is why we examine both one and two arcminute radius regions, and why some fields may have an overdensity within two arcminutes but not within one arcminute. Figures 3 and 4 compare the source count distributions over all the COBRA fields to those measured with an identical procedure but in the SpUDS mosaics.

Candidate galaxy clusters were selected as those having either an overdensity of $\geq 2 \sigma$ within $1^{\prime}$ (an excess of 12.4 sources) or an overdensity of $\geq 2 \sigma$ within $2^{\prime}(29.4$ sources), or an excess of sources within both radii, to a limiting magnitude of $m_{3.6}=21.4$.

We calculated the error and significance of our overdensities as follows. First, to determine the error on the overdensity, we assumed Poisson statistics, including the counts in the field surrounding each radio source and the background counts as determined from the SpUDS field, and calculated the error on $\Delta N, \sigma_{\Delta N}$ (columns 10 and 13 in Table 1), with the following equation:

$$
\sigma_{\Delta N}=\sqrt{\Delta N+A\left(1+A / A_{b k g}\right) \Sigma_{a v g}} .
$$

In Equation 1, $\Delta N$ is the number of detected sources above the mean background, $A$ is the area of the COBRA field in which sources were counted, $A_{b k g}$ is the area of the entire SpUDS field within which sources were counted, and $\Sigma_{a v g}$ is the average surface density of the SpUDS field. Second, we use the sources detected around each radio source and the one-sigma variance of the Gaussian fit to determine the significance (columns 10 and 13 in Table 1) using the following equation:

$$
\text { Significance }=\frac{\Delta \mathrm{N}}{\sigma_{\mathrm{SpUDS}}} .
$$

This is the number of detected sources above the mean background divided by the standard deviation of the Gaussian fit.

Table 1 shows the results of our search. Column 1 lists the name of the source, while Columns 2 and 3 list the coordinates of the optical/IR host (see $\S 5$ for a 


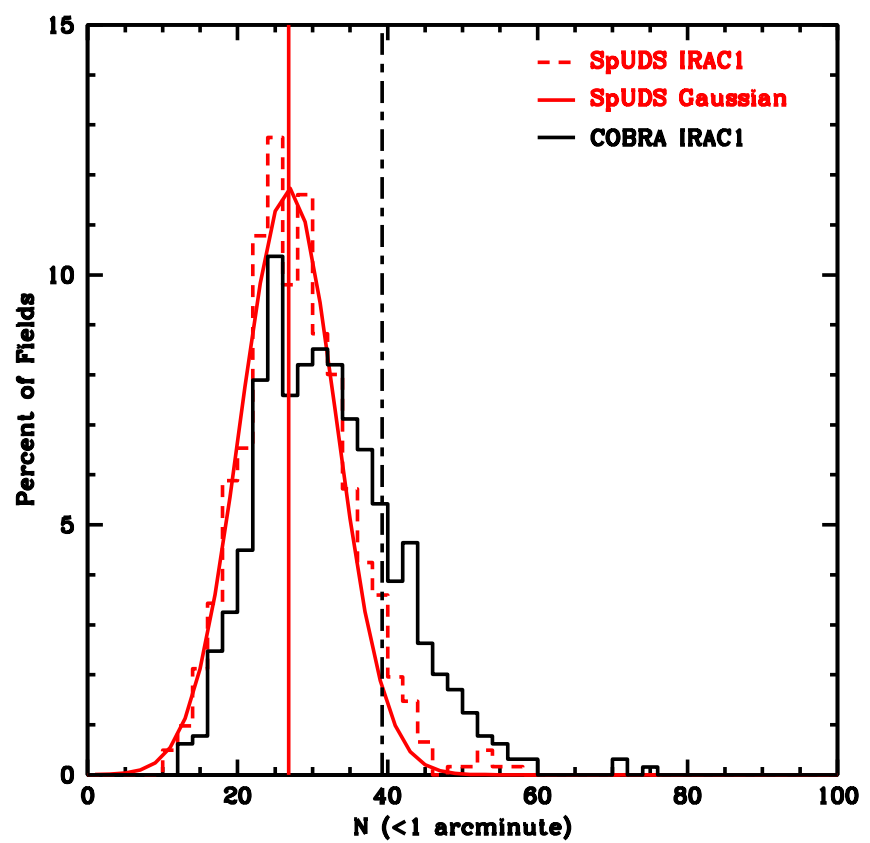

Figure 3. Histogram of the percentage of fields with a given number of detected sources in a one arcminute radius region for a limiting magnitude of $m_{3.6}=21.4$. The solid black histogram shows results from the 646 COBRA fields, while the dashed red histogram shows results from the 612 regions in the SpUDS field. The solid, red curve shows the Gaussian fit described in $\S 4.1$. The solid, red vertical line shows the mean of the Gaussian fit, and the dot-dash, black vertical line marks an excess of 12.4 sources, which corresponds to an overdensity of $2 \sigma$. Objects to the right of this line are cluster candidates. detailed discussion of how the optical host coordinates were obtained). Column 4 lists the $20 \mathrm{~cm}$ flux density as measured by FIRST and Column 5 lists the magnitude of the host in the $3.6 \mu \mathrm{m}$ band as determined by SExtractor. Where available, host color $\left(m_{3.6}-m_{4.5}\right)$ and redshift are listed in columns 6 and 7 . Columns $8-10$ give the results from the search within a one arcminute radius, while columns $11-13$ give the results from the search within a two arcminute radius to a limiting magnitude of $m_{3.6}=21.4$. 


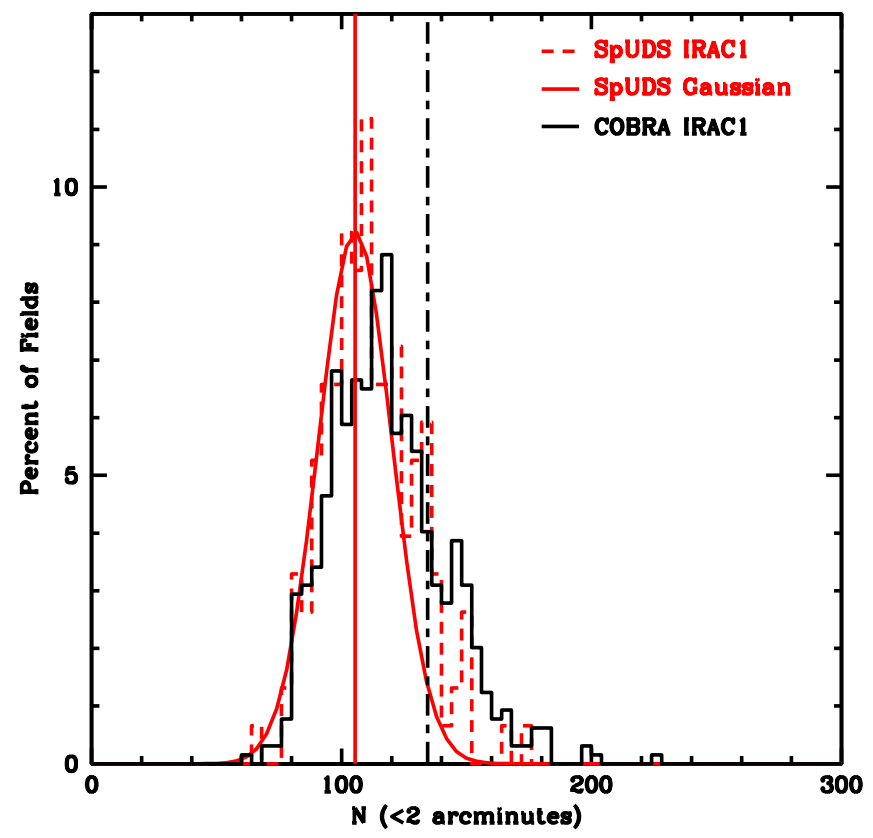

Figure 4. Histogram of the percentage of fields with a given number of detected sources in a two arcminute radius region to a limiting magnitude of $m_{3.6}=21.4$. The solid black histogram shows results from the 646 COBRA fields, while the dashed red histogram shows results from the 152 regions in the SpUDS field. The solid, red curve shows the Gaussian fit described in $\S 4.1$. The solid, red vertical line shows the mean of the Gaussian fit, and the dot-dash, black vertical line marks an excess of 29.4 sources, which corresponds to an overdensity of $2 \sigma$. Objects to the right of this line are cluster candidates. 


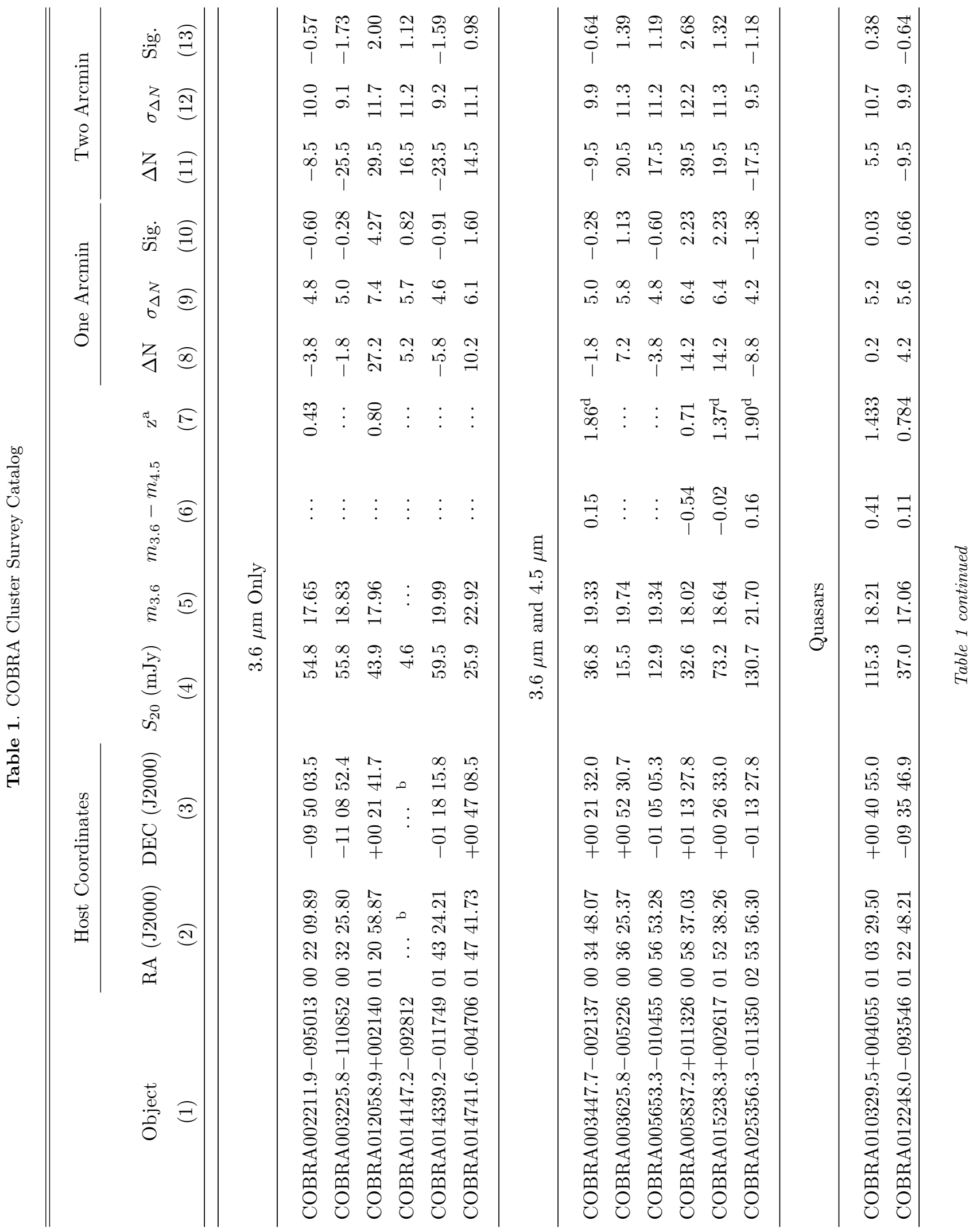




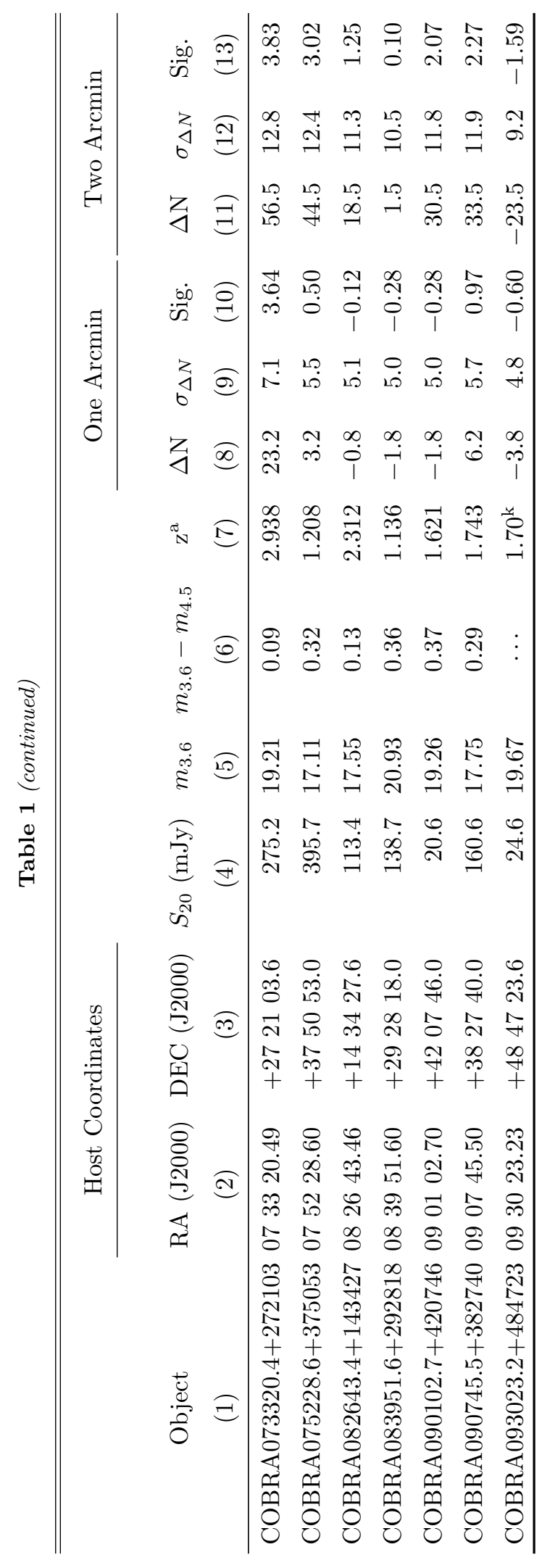




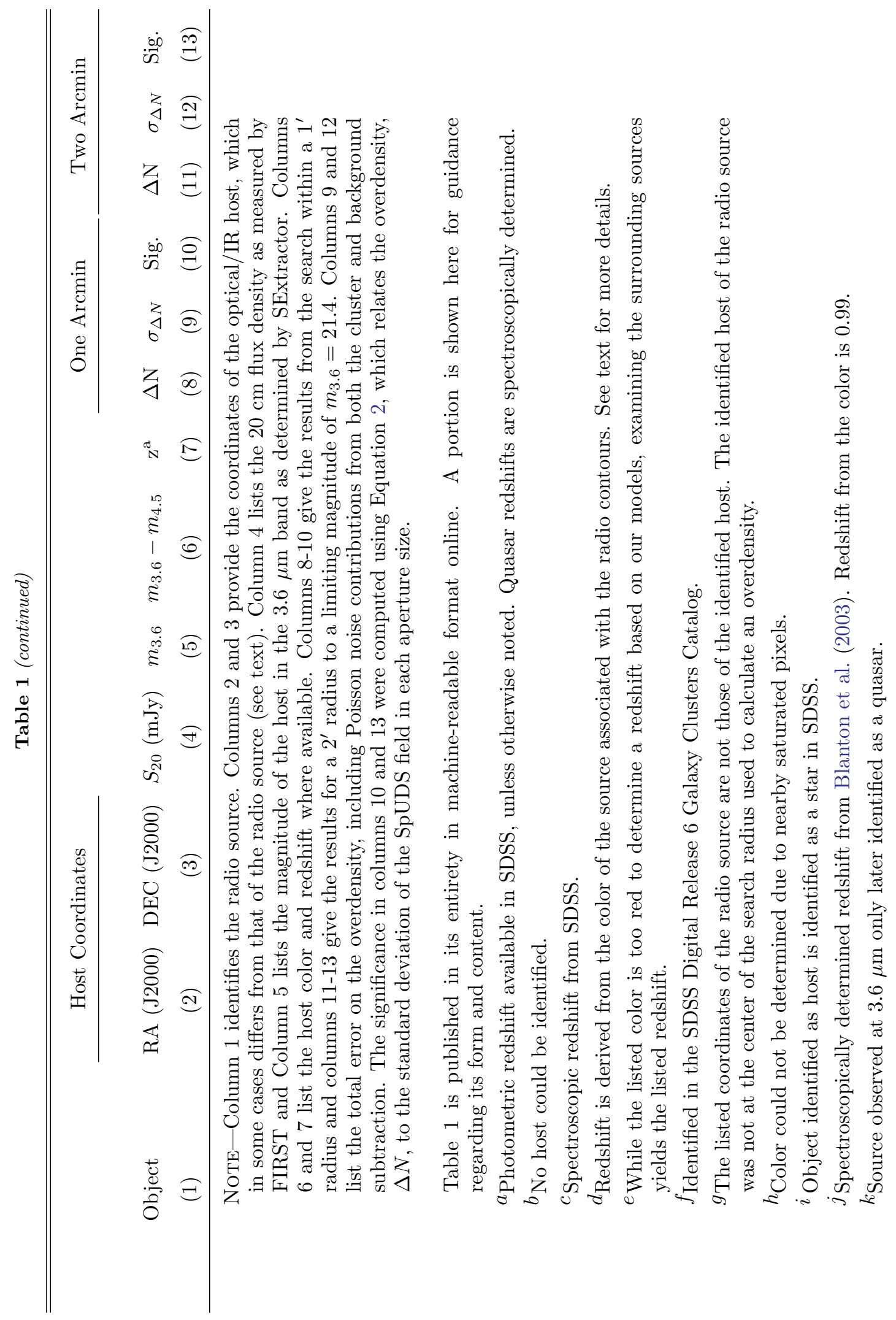


Table 2. Number of Cluster Candidates

\begin{tabular}{lccccc}
\hline \hline \multicolumn{1}{c}{ Sample } & $1^{\prime}$ Only & $2^{\prime}$ Only & Both & Total & $\%$ \\
\hline All COBRA & 48 & 47 & 95 & 190 & 29.4 \\
Non-quasars & 48 & 39 & 90 & 177 & 29.3 \\
Quasars & 0 & 8 & 5 & 13 & 31.7 \\
\hline
\end{tabular}

Note-Column 1 identifies the subsample. Columns 24 provide the number of cluster candidates within the different search radii. Column 5 gives the total number of cluster candidates for each subsample. Column 6 is the percentage of the targets in the subsample that are cluster candidates.

To a limiting magnitude of $m_{3.6}=21.4$ we identify 48 fields that only satisfy the $1^{\prime}$ overdensity criterion, 47 that only satisfy the $2^{\prime}$ criterion, and 95 which met both criteria. In total, 190 fields meet at least one of the two overdensity criteria with a limiting magnitude of $m_{3.6}=21.4$, giving a total cluster association rate of $29.4 \%$ to our adopted limiting magnitude.

Figure 5 shows the distribution of the overdensities surrounding each bent AGN to a limiting magnitude of $m_{3.6}=21.4$. The majority of the cluster candidates show overdensities within both search radii (defined as $\Delta N \geq 12.4$ within one arcminute and $\Delta N \geq 29.4$ within two arcminutes). This is also true of the quasars. Excluding all the sources identified as quasars, there are 48 radio sources that are surrounded by overdensities within one arcminute only, 39 radio sources that are surrounded by overdensities within two arcminutes only, and 90 radio sources that are surrounded by overdensities within both search radii.

Of the 41 quasars that meet our selection criteria, 13 are cluster candidates, for an association rate of $31.7 \%$. This is comparable to the association rate of the nonquasars $(29.3 \%)$. The five that are cluster candidates within one arcminute also satisfy the candidate selection criterion within two arcminutes. There are an additional eight cluster candidates that are not overdense within one arcminute but are within two arcminutes. This is tentative evidence that quasars tend to reside on the outskirts of clusters, which is to be expected if they are triggered during infall as clusters build up. Further observations will be helpful in testing this hypothesis, which is very preliminary. Golden-Marx et al. (in preparation) will explore the relationship between the the radio source position and the projected surface density of sources and will compare the quasar/non-quasar differences in more detail. Table 2 summarizes the number of cluster candidates found in each subsample.

There are 376 COBRA fields (including the quasars) with $\Delta N \geq 0$ within both search radii, 77 with $\Delta N \geq 0$

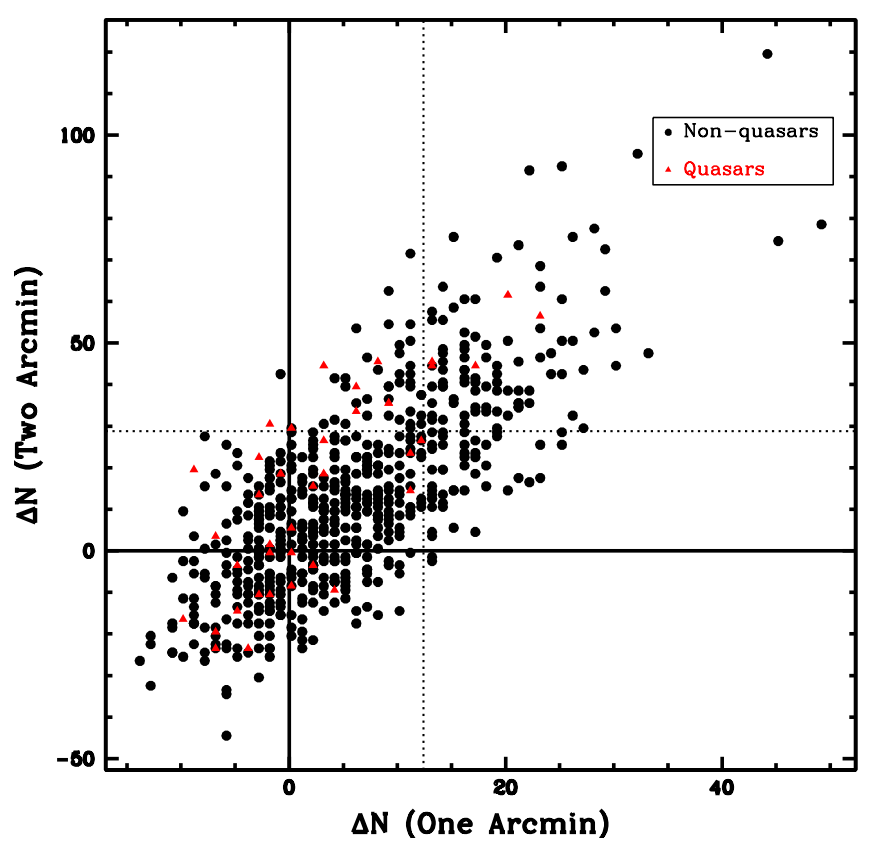

Figure 5. Distribution of excess sources above background within two arcmin plotted against the excess above background within one arcmin in the COBRA fields to a limiting magnitude of $m_{3.6}=21.4$. The overdensities of fields surrounding quasars are plotted as red triangles, while the overdensities of fields around non-quasars are plotted as black circles. The vertical dashed line marks an overdensity of $2 \sigma$ within one arcmin (i.e. anything to the right would be considered a cluster searching within a one arcmin radius region) and the horizontal dashed line marks an overdensity of $2 \sigma$ within two arcmin (i.e. anything above that line would be a considered a cluster with a search radius of two arcmin). $\Delta N=0$ indicates that the number of sources surrounding the AGN is equal to the mean background value. Any $\Delta N>0$ indicates an excess above the mean background (solid black lines). Due to the scaling of the axes, some of the points for the quasars overlap with each other.

within one arcminute but with underdensities $(\Delta N<0)$ within two arcminutes, and 77 with $\Delta N \geq 0$ within two arcminutes but underdensities within one arcminute, for a total of 530 fields $(82.0 \%)$ that have an excess number of sources as compared to the background. There are 4 quasars that have $\Delta N \geq 0$ within one arcminute but are underdense within two arcminutes, and 9 more that have $\Delta N \geq 0$ within two arcminutes but are underdense within one arcminute. There are an additional 18 quasars that have $\Delta N \geq 0$ within both search radii. These results are summarized in Table 3 . It is likely that the targets that only have overdensities in the larger search radius are not as centrally peaked, and are in the process of merging. Alternatively, the bent radio source 
Table 3. Number of Targets with Positive Overdensities $(\Delta N \geq 0)$

\begin{tabular}{lccccc}
\hline \hline \multicolumn{1}{c}{ Sample } & $1^{\prime}$ Only & $2^{\prime}$ Only & Both & Total & $\%$ \\
\hline All COBRA & 77 & 77 & 376 & 530 & 82.0 \\
Non-Quasars & 73 & 68 & 358 & 499 & 82.5 \\
Quasars & 4 & 9 & 18 & 31 & 75.6 \\
\hline
\end{tabular}

Note-Column 1 identifies the subsample. Columns 2-4 provide the number of targets with positive overdensities within the different search radii. Column 5 gives the total number of targets with positive overdensities for each subsample. Column 6 is the percentage of targets in that subsample with positive overdensities.

may just be located away from the center of a (possibly relaxed) cluster.

\section{REDSHIFT ESTIMATES}

For the COBRA targets that were observed in both the $3.6 \mu \mathrm{m}$ and $4.5 \mu \mathrm{m}$ bands, we used the color of the radio host to estimate a preliminary redshift. To find the host galaxy, we performed a radial search (expanding in radius until a match was returned) around the coordinates of the radio source as defined in $\S 2$, assuming that the closest match was the host. We then visually inspected the radio contours overlaid on the Spitzer images to determine if there was a better match. If there was a better match, its coordinates were obtained by searching the SExtractor catalog for the nearest SExtractor source detected within $2^{\prime \prime}$ of the coordinates of the new host. The SDSS was searched again for redshifts after all hosts were identified. Updated host coordinates are listed in Columns 2 and 3 of Table 1. All of the 32 original quasars have optical hosts at the same coordinates (to within $2^{\prime \prime}$ ) as the radio coordinates. There are 15 non-quasars whose optical/infrared host could not be identified. Once the host was determined, we used the galaxy model code EzGal (Mancone \& Gonzalez 2012) with a Salpeter initial mass function, metallicity of 0.02 , and a Bruzual \& Charlot (2003) simple stellar population model with a formation redshift of $z=3$ to relate our measured colors to redshifts. Based on the limiting magnitude of SDSS, we assume that all host galaxies have a redshift $z>0.7$, unless otherwise noted. At $z \gtrsim 1.4$ the relation between color and redshift is not monotonic (Figure 6), and occasionally there were two solutions for a given color. For those hosts we list only the lower redshift.

Some sources in Table 1 have either spectroscopic or photometric redshifts below our original redshift cutoff estimate of $z \approx 0.7$. These sources were not found in the

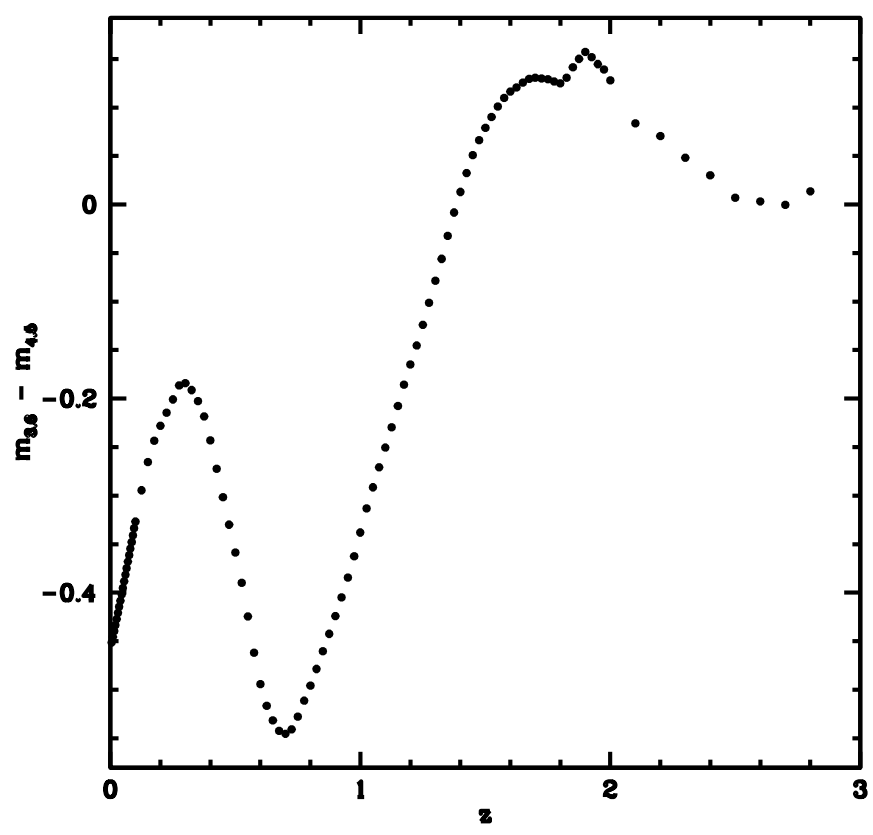

Figure 6. Color $\left(m_{3.6}-m_{4.5}\right)$ as a function of redshift for a galaxy model with a Salpeter initial mass function, metallicity of 0.02, and a Bruzual \& Charlot (2003) simple stellar population model with a formation redshift of $z=3$.

original search by Wing \& Blanton (2011) due to either large offsets in the position of the estimate of the radio source core or the magnitude limit cutoff of $m_{r}=22$ in the original search. Here we do not impose a magnitude limit in the SDSS. Additionally, while the radio galaxy hosts are often giant ellipticals, they can have a wide range of luminosities and thus it is possible some have magnitudes fainter than $m_{r}=22$ and redshifts $z<0.7$. The core of the radio source was determined visually in the visual-bent sample; however in the auto-bent sample the coordinates of the core were determined to be the component opposite the longest side when making a triangle of the three components. After inspection of the Spitzer images, the host galaxy was sometimes found to be associated with another radio component.

The majority of the redshifts determined from the infrared color of the source are consistent with the redshift listed in SDSS (within errors, which are $\sim 10 \%$ ). For the few that are not, we keep the SDSS redshift, as they have more filters and thus a more accurate photometric redshift.

Figure 7 shows the redshift distribution of all IRACdetected radio hosts in the COBRA fields that were observed in both bands $(3.6 \mu \mathrm{m}$ and $4.5 \mu \mathrm{m})$ for which we were able to estimate redshifts. We include all the sources (dashed black line); we also separately show the distribution for our candidate clusters (solid red line). All of the quasars in our sample have redshifts greater than $z=0.7 ; 13$ of the regions surrounding the quasars 


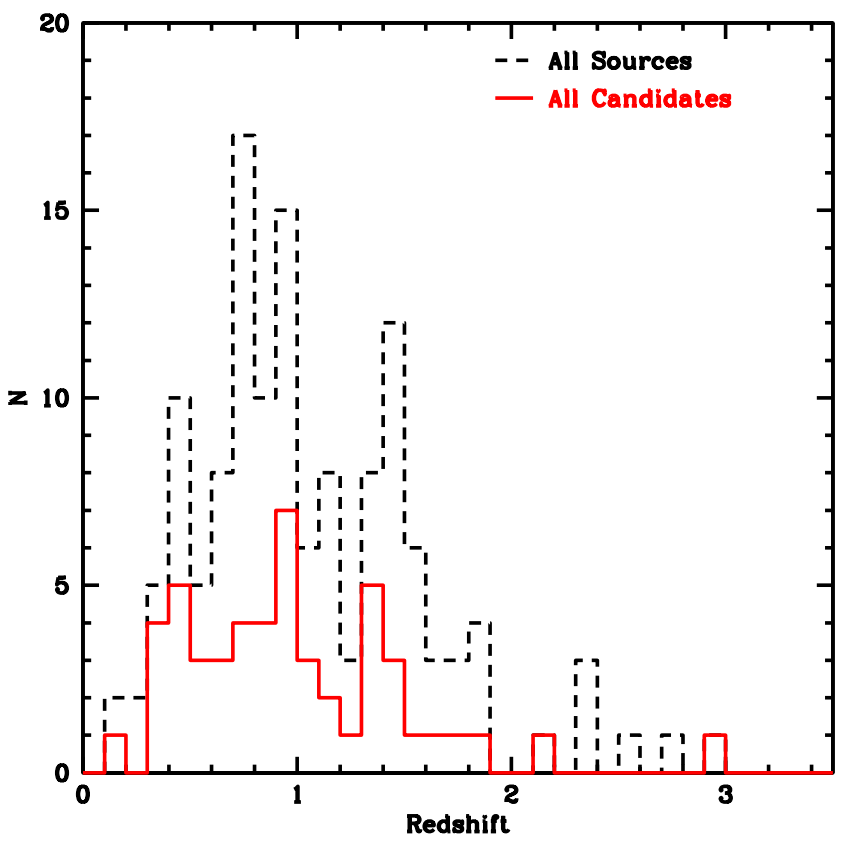

Figure 7. Histogram of the redshift distribution of all sources that were observed in both the $3.6 \mu \mathrm{m}$ and $4.5 \mu \mathrm{m}$ bands with an estimated (or known, in the case of the quasars and sources found in the SDSS) redshift. The dashed black line includes all sources; the solid red line shows the distribution of sources identified as cluster candidates. For sources with degenerate redshift estimates, we used the lower one for the histogram. Our objects extend in redshift up to $z \approx 3.0$.

were identified as cluster candidates. There are 103 non-quasar sources observed in both bands. Of these, 41 were identified as cluster candidates. We were only able to estimate redshifts for 94 of the 103 non-quasar sources; the remaining sources were either too red to use the normal galaxy color-redshift relation or did not have an identifiable host. Three of these nine are cluster candidates. Of the 38 non-quasar cluster candidates with redshifts, 22 have $z>0.7,7$ have $0.5 \leq z<0.7$, and 9 have $z<0.5$. All of the ones with redshifts $z<0.7$ were either spectroscopically or photometrically identified in the SDSS.

\section{INDIVIDUAL CLUSTERS}

We have selected six examples (Figure 8) of our candidate clusters to discuss in more detail. We will discuss one source for which a redshift could not be estimated, two with estimated but unconfirmed redshifts, one source observed in both bands that also has a spectroscopically confirmed redshift in SDSS, and two quasars, which have known redshifts. All of the sources show clear bent morphology and have moderate-to-high overdensities. For those sources with redshifts, we also discuss the extent of the radio source (as measured from the edge of one lobe to the edge of the other) and the radio luminosity of the source. For the five of these six radio sources for which we have redshifts, we can calculate the radio luminosity of the source using the following equation:

$$
L_{\text {rad }}=4 \pi D_{L}^{2} S_{\nu_{0}} \int_{\nu_{1}}^{\nu_{2}}\left(\frac{\nu}{\nu_{0}}\right)^{-\alpha} d \nu
$$

where $D_{L}$ is the luminosity distance, $S_{\nu_{0}}$ is the total flux density at the reference frequency $\nu_{0}$, and $\alpha$ is the spectral index. For all our sources, we assume a spectral index $\alpha=0.8$, as is typical for extragalactic radio sources (Sarazin 1988). We take $1400 \mathrm{MHz}$ as our reference frequency, corresponding to the average frequency where FIRST measured the radio flux densities for our sources, and use the integration limits of $10^{7} \mathrm{~Hz}$ to $10^{11} \mathrm{~Hz}$, taking into account the redshift of each source. The radio flux density for each source was measured at $20 \mathrm{~cm}$ by FIRST.

The candidate clusters in this survey, including those discussed below, are being imaged in the optical with the Discovery Channel Telescope (DCT) as part of a comprehensive follow-up program to study these objects in more detail. The DCT is a 4.3 meter telescope located in Happy Jack, AZ. These objects are being observed (Golden-Marx et al., in preparation) with the Large Monolithic Imager, a single chip CCD with a 12 '. $3 \times 12$ '.3 field of view.

COBRA091928.9+273215: COBRA091928.9+273215 was observed in both bands, and is an example of a moderately overdense region. It has an excess of 19.2 sources within $1^{\prime}$ and an excess of 44.5 sources within $2^{\prime}$. This corresponds to a Gaussian overdensity of $3.0 \sigma$ within both radii. This source has a photometric redshift of $z=0.63$ in the SDSS. The radio lobes extend $50^{\prime \prime} .8$, which corresponds to $347 \mathrm{kpc}$ at this redshift. Its radio luminosity is $L_{r a d}=1.35 \times 10^{43} \mathrm{erg} \mathrm{s}^{-1}$, using a luminosity distance $D_{L}=3745.7 \mathrm{Mpc}$ and a $20 \mathrm{~cm}$ radio flux density of $S_{0}=52.9 \mathrm{mJy}$.

COBRA 104254.8+290719: COBRA104254.8+290719 was observed in both bands. It has an excess within $1^{\prime}$ of 22.2 sources, which corresponds to a Gaussian significance of $3.5 \sigma$. It is not overdense within $2^{\prime}$. Because we had observations in both bands, we used the color of the host to estimate a preliminary redshift, as discussed in $\S 5$. We find that this source has an approximate redshift of $z=1.04$. It extends 54"'3, which corresponds to $438.7 \mathrm{kpc}$ at this redshift. Its radio luminosity is $L_{\text {rad }}=3.06 \times 10^{43} \mathrm{erg} \mathrm{s}^{-1}$, using a luminosity distance $D_{L}=6935.5 \mathrm{Mpc}$ and a $20 \mathrm{~cm}$ radio flux density of $S_{0}=33.3 \mathrm{mJy}$. This candidate has DCT follow-up observations, which show overdensities consistent with those observed with Spitzer (Golden-Marx et al., in preparation).

COBRA141155.2+341510: COBRA141155.2+341510 is a quasar with redshift of 1.82 and has an overdensity within both one and two arcminutes. Within a $1^{\prime}$ radius (506 kpc), it has an excess of 17.2 sources, which 

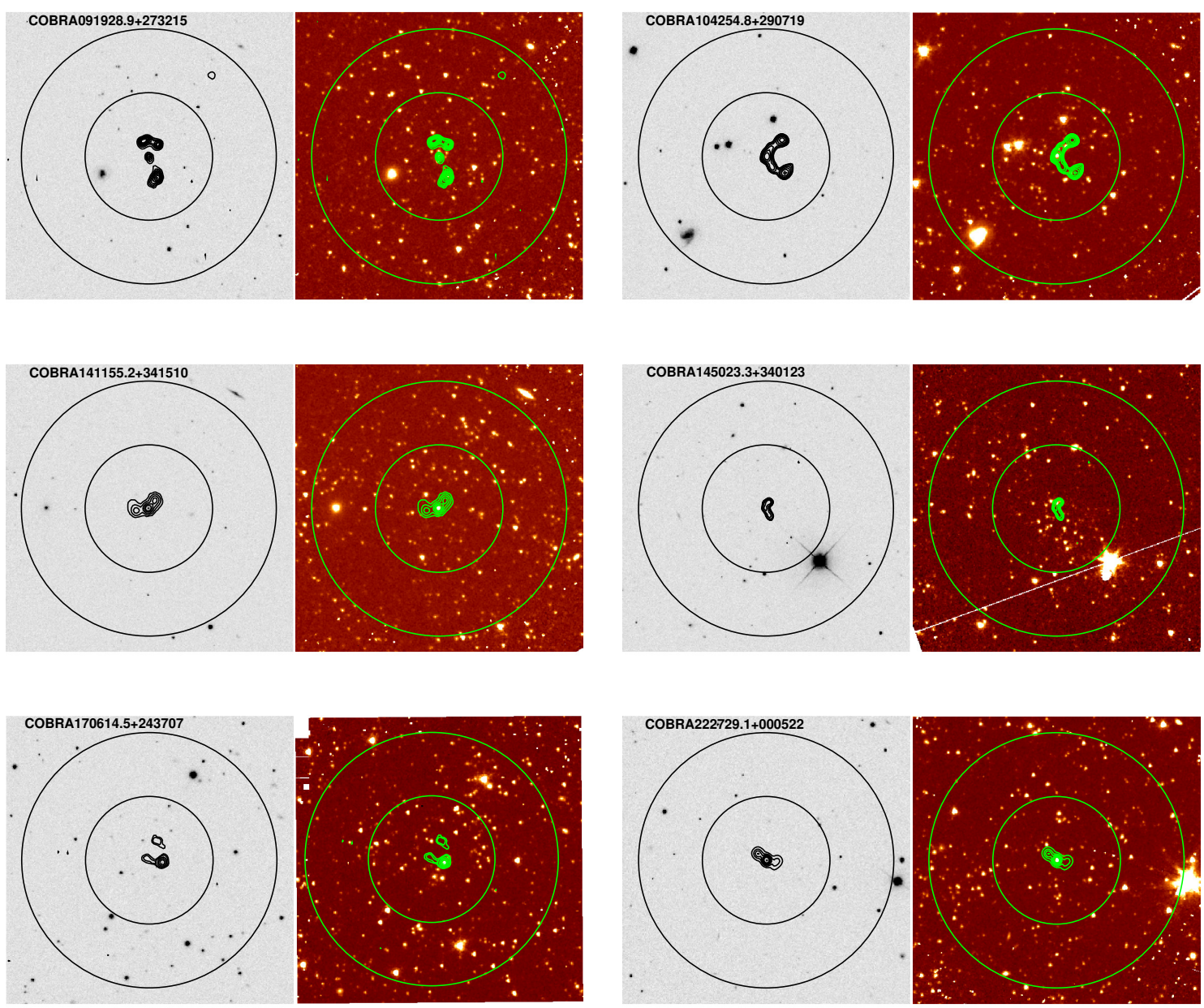

Figure 8. Montage showing the SDSS $r$-band and IRAC $3.6 \mu \mathrm{m}$ mosaics of six representative COBRA cluster candidates. Greyscale: SDSS-r image of the area around the radio source. Orange: Spitzer $3.6 \mu \mathrm{m}$ image. The VLA FIRST radio contours are overlaid on both panels. The inner circle in both panels is one arcminute in radius, while the outer circle is two arcminutes in radius. The radio host is not visible in the SDSS-r band, but becomes visible in the $3.6 \mu \mathrm{m}$ Spitzer image, along with additional sources. In all panels, north is up and east is to the left.

corresponds to $2.7 \sigma$ when compared to the Gaussian fit of the background. Within a $2^{\prime}$ radius the overdensity increases to $3.0 \sigma$, which corresponds to 44.5 sources above the background (the overdensity can be seen to the northwest of the radio source). As mentioned in $\S 4.2$, quasars may be triggered during infall, and thus reside on the outskirts of clusters. It is possible that we are seeing this here. While the quasar is visible in the SDSS-r image, the majority of the other sources are not. The lobes of the quasar are $355^{\prime \prime} 8$ (709 kpc) in extent. The total radio luminosity is $L_{\text {rad }}=7.81 \times 10^{44} \mathrm{erg} \mathrm{s}^{-1}$, using a luminosity distance of $D_{L}=13840.7 \mathrm{Mpc}$ and a reference radio flux density of $S_{0}=200.1 \mathrm{mJy}$. This source has been observed with the DCT and shows an overdensity in the deep optical images, as well.

COBRA145023.3+340123: COBRA145023.3+340123 is an example of an highly overdense target within $1^{\prime}$ but not within $2^{\prime}$. It was observed at $3.6 \mu \mathrm{m}$ only, although it has been observed at optical wavelengths with the DCT. It has an excess of 23.2 sources within $1^{\prime}$ which corresponds to an overdensity of $3.6 \sigma$. The extent of the radio source is $233^{\prime \prime} 2$. If we assume a redshift of $z=1.0$ (the approximate peak of the redshift distribution shown in Figure 7 ), this corresponds to $185.8 \mathrm{kpc}$.

COBRA170614.5+243707: COBRA170614.5+243707 is another highly overdense region in our sample. It was observed in both bands, and has a redshift of $z=0.71$, which was confirmed spectroscopically in SDSS. It has an excess of 29.2 sources within a $1^{\prime}$ region, which corresponds to a significance of $4.6 \sigma$. Within $2^{\prime}$ the excess is 72.5 sources, which corresponds to a Gaussian significance of $4.9 \sigma$. The radio lobes extend $46^{\prime \prime}(330.6 \mathrm{kpc})$. The total radio luminosity of this 
source is $L_{\text {rad }}=6.37 \times 10^{42} \mathrm{erg} \mathrm{s}^{-1}$. This was calculated using a luminosity distance $D_{L}=4334.1 \mathrm{Mpc}$ and a reference flux density $S_{0}=18.4 \mathrm{mJy}$. This source has been observed with the DCT.

COBRA222729.1+000522: COBRA222729.1+000522 is a quasar with a redshift of 1.513 . It is overdense within both $1^{\prime}(508 \mathrm{kpc})$ and $2^{\prime}$. Within $1^{\prime}$ there is an excess of 13.2 sources, which corresponds to a significance of $2.1 \sigma$. Within $2^{\prime}$, there is an excess of 45.5 sources, which corresponds to a significance of $3.1 \sigma$. As with J141155.2+341510, the stronger overdensity within $2^{\prime}$ than $1^{\prime}$ suggests that the quasar resides on the cluster outskirts. The lobes of the quasar extend $30^{\prime \prime} 9(261.5 \mathrm{kpc})$, and it has a radio luminosity $L_{\text {rad }}=4.32 \times 10^{44} \mathrm{erg} \mathrm{s}^{-1}$. The radio luminosity was calculated using a luminosity distance $D_{L}=11025.2 \mathrm{Mpc}$ and a reference flux density $S_{0}=178.6 \mathrm{mJy}$. This source has been observed with the DCT.

\section{DISCUSSION AND CONCLUSIONS}

The high-redshift COBRA survey consists of 646 bent, double-lobed radio sources observed with Spitzer in the $3.6 \mu \mathrm{m}$ band (135 of these 646 sources were also observed at $4.5 \mu \mathrm{m})$. Forty-one of these sources are quasars with spectroscopic redshifts above $z=0.7$; the range extends to $z \approx 3$. We compared the number of sources as determined by running SExtractor on the region around the radio source to the expected number of sources based on the surface density of the SpUDS field. We found 190 fields with overdensities corresponding to at least a $2 \sigma$ significance. These 190 over-dense regions are likely clusters, most of them at high redshifts. Of the 190 cluster candidates, 39 have $z<0.5,32$ have $0.5 \leq z<0.7$ and 119 have $z \geq 0.7$ or do not have a known redshift, indicating that they are likely to have $z \geq 0.7$. We will explore the relationship between overdensity and redshift in a forthcoming paper.

Wylezalek et al. (2013) performed a similar analysis using obscured and unobscured powerful radio-loud (but not necessarily bent) AGN in the redshift range $1.2<z<3.2$ (the Clusters Around Radio-Loud AGN, or CARLA, sample) and found that $92 \%$ of sources reside in a denser-than-average region. They also find that the majority $(55 \%)$ of their sources reside in regions that are overdense at at least the $2 \sigma$ level. To directly compare their results with ours, we ran their images through our pipeline, using the same parameters and methodology as described in $\S 3.2$ and $\S 4$. Doing so, we find that $43.9 \%$ of the CARLA fields are overdense at the $2 \sigma$ level or higher and $88.1 \%$ of the CARLA fields have a positive excess of galaxies as compared to the background. At a $2 \sigma$ or higher confidence level, we find that $29.4 \%$ of our fields are overdense, while $82.0 \%$ of our fields have a positive excess of galaxies as compared to the background. Two sources, COBRA073320.4+272103 and COBRA143331.9+190711, appear in both surveys. Both objects are quasars. COBRA073320.4+272103 is found to be very overdense $(>3.5 \sigma)$ in both surveys, while COBRA143331.9+190711 has a positive excess of galaxies in both surveys but is not overdense at the $\geq 2 \sigma$ level. Both surveys are finding overdense regions at a similar rate. The mean radio power of the sources in the CARLA sample is higher than in our COBRA sample. These powerful, radio-loud AGN tend to be found in somewhat richer environments on average (Hatch et al. 2014). Bent sources are found in a wide range of environments, including clusters, groups, and even large-scale filaments (Edwards et al. 2010).

Using a somewhat different method, in another study, Galametz et al. (2012) find that $73 \%$ of their radio sources reside in regions with overdensities of 15 or more sources within one arcminute. All of these surveys show that regardless of morphology, radio AGN are good tracers of high-redshift clusters.

At low redshift, bent, double-lobed radio AGN are found in both relaxed (A2029, Clarke et al. 2004; Paterno-Mahler et al. 2013) and merging (A562, Douglass et al. 2011) clusters. Simpson \& Rawlings (2002) suggest that at redshifts $1<z<2$ radio AGN may be triggered by galaxy-galaxy mergers that are most likely triggered by cluster mergers. As Brodwin et al. (2013) discuss, the merging causes a burst of star formation and fuels an AGN that eventually quenches the star formation by heating up the cold gas and/or expelling it. Such merging may also explain the rapid mass growth observed by Mancone et al. (2010), the high AGN incidence observed by Martini et al. (2013) and Galametz et al. (2009), the scatter in cluster red sequences and young ages of red sequence galaxies observed by Snyder et al. (2012), as well as many other measurements. These galaxy-galaxy mergers can also happen in lowermass clusters whose smaller velocity dispersions lead to increased merger efficiency. Such clusters need not be disturbed by cluster-scale mergers. Recently Cooke et al. (2016) identified a high-redshift $(z=1.58)$ relaxed cluster selected using a powerful radio AGN as a signpost, indicating that such sources are found in a variety of environments at both high- and low-redshift. AGN may also be triggered by inflowing gas in relaxed, cool core clusters(McNamara \& Nulsen 2007). Our sample will be sensitive to both merging and relaxed groups and clusters, and thus we will be able to explore galaxy evolution in both environments. Combined with the wide range of masses we are probing, we will explore how these properties affect galaxy evolution in ongoing work.

First and foremost, we thank the referee for a thorough and valuable review.

We thank the CARLA team for generously sharing their data with us.

RPM would like to thank Michael Malmrose for useful discussion. 
This work has been supported by the National Science Foundation, grant AST-1309032.

This work is based in part on observations made with the Spitzer Space Telescope, which is operated by the Jet Propulsion Laboratory, California Institute of Technology under a contract with NASA. Support for this work was provided by NASA through an award issued by JPL/Caltech (NASA award RSA No. 1440385).

Funding for SDSS-III has been provided by the Alfred P. Sloan Foundation, the Participating Institutions, the National Science Foundation, and the U.S. Department of Energy Office of Science. The SDSS-III web site is http://www.sdss3.org/.

SDSS-III is managed by the Astrophysical Research Consortium for the Participating Institutions of the SDSS-III Collaboration including the University of Ari- zona, the Brazilian Participation Group, Brookhaven National Laboratory, Carnegie Mellon University, University of Florida, the French Participation Group, the German Participation Group, Harvard University, the Instituto de Astrofisica de Canarias, the Michigan State/Notre Dame/JINA Participation Group, Johns Hopkins University, Lawrence Berkeley National Laboratory, Max Planck Institute for Astrophysics, Max Planck Institute for Extraterrestrial Physics, New Mexico State University, New York University, Ohio State University, Pennsylvania State University, University of Portsmouth, Princeton University, the Spanish Participation Group, University of Tokyo, University of Utah, Vanderbilt University, University of Virginia, University of Washington, and Yale University.

Facilities: Spitzer, Sloan

\section{REFERENCES}

Abazajian, K. N., Adelman-McCarthy, J. K., Agüeros, M. A., et al. 2009, ApJS, 182, 543

Abell, G. O. 1958, ApJS, 3, 211

Ascasibar, Y., \& Markevitch, M. 2006, ApJ, 650, 102

Ashby, M. L. N., Willner, S. P., Fazio, G. G., et al. 2015, ApJS, 218, 33

Bahcall, N. A. 1975, ApJ, 198, 249

Becker, R. H., White, R. L., \& Helfand, D. J. 1995, ApJ, 450, 559

Bertin, E., \& Arnouts, S. 1996, A\&AS, 117, 393

Blanton, E. L. 2000, PhD thesis, COLUMBIA UNIVERSITY

Blanton, E. L., Gregg, M. D., Helfand, D. J., Becker, R. H., \& Leighly, K. M. 2001, AJ, 121, 2915

Blanton, E. L., Gregg, M. D., Helfand, D. J., Becker, R. H., \& White, R. L. 2000, ApJ, 531, 118

-. 2003, AJ, 125, 1635

Bleem, L. E., Stalder, B., de Haan, T., et al. 2015, ApJS, 216, 27

Brodwin, M., Brown, M. J. I., Ashby, M. L. N., et al. 2006, ApJ, 651, 791

Brodwin, M., Stanford, S. A., Gonzalez, A. H., et al. 2013, ApJ, 779, 138

Brodwin, M., Greer, C. H., Leitch, E. M., et al. 2015, ApJ, 806, 26

Bruzual, G., \& Charlot, S. 2003, MNRAS, 344, 1000

Burns, J. O. 1990, AJ, 99, 14

Churazov, E., Vikhlinin, A., \& Sunyaev, R. 2015, MNRAS, 450, 1984

Clarke, T. E., Blanton, E. L., \& Sarazin, C. L. 2004, ApJ, 616,178

Cooke, E. A., Hatch, N. A., Stern, D., et al. 2016, ApJ, 816,83
Dehghan, S., Johnston-Hollitt, M., Franzen, T. M. O., Norris, R. P., \& Miller, N. A. 2014, AJ, 148, 75

Douglass, E. M., Blanton, E. L., Clarke, T. E., Randall, S. W., \& Wing, J. D. 2011, ApJ, 743, 199

Dressler, A. 1978, ApJ, 226, 55

Edwards, L. O. V., Fadda, D., \& Frayer, D. T. 2010, ApJL, 724, L143

Eisenhardt, P. R. M., Brodwin, M., Gonzalez, A. H., et al. 2008, ApJ, 684, 905

Fazio, G. G., Hora, J. L., Allen, L. E., et al. 2004, ApJS, 154,10

Galametz, A., Stern, D., Eisenhardt, P. R. M., et al. 2009, ApJ, 694, 1309

Galametz, A., Stern, D., De Breuck, C., et al. 2012, ApJ, 749,169

Giacintucci, S., \& Venturi, T. 2009, A\&A, 505, 55

Gladders, M. D., \& Yee, H. K. C. 2000, AJ, 120, 2148

Gonzalez, A. H., Decker, B., Brodwin, M., et al. 2015, ApJL, 812, L40

Hasselfield, M., Hilton, M., Marriage, T. A., et al. 2013, JCAP, 7, 008

Hatch, N. A., Wylezalek, D., Kurk, J. D., et al. 2014, MNRAS, 445, 280

Hennig, C., Mohr, J. J., Zenteno, A., et al. 2016, ArXiv e-prints

Lacy, M., Wilson, G., Masci, F., et al. 2005, ApJS, 161, 41

Lakhchaura, K., Singh, K. P., Saikia, D. J., \& Hunstead,

R. W. 2011, ApJ, 743, 78

Lin, Y.-T., \& Mohr, J. J. 2007, ApJS, 170, 71

Mancone, C. L., \& Gonzalez, A. H. 2012, PASP, 124, 606

Mancone, C. L., Gonzalez, A. H., Brodwin, M., et al. 2010, ApJ, 720, 284 
Marriage, T. A., Acquaviva, V., Ade, P. A. R., et al. 2011, ApJ, 737, 61

Martini, P., Miller, E. D., Brodwin, M., et al. 2013, ApJ, 768,1

McGee, S. L., Balogh, M. L., Bower, R. G., Font, A. S., \& McCarthy, I. G. 2009, MNRAS, 400, 937

McNamara, B. R., \& Nulsen, P. E. J. 2007, ARA\&A, 45, 117

Miley, G., \& De Breuck, C. 2008, A\&A Rv, 15, 67

Muzzin, A., Wilson, G., Lacy, M., Yee, H. K. C., \& Stanford, S. A. 2008, ApJ, 686, 966

Muzzin, A., Wilson, G., Yee, H. K. C., et al. 2009, ApJ, 698, 1934

Norris, R. P. 2011, Journal of Astrophysics and Astronomy, 32,599

Papovich, C. 2008, ApJ, 676, 206

Paterno-Mahler, R., Blanton, E. L., Randall, S. W., \& Clarke, T. E. 2013, ApJ, 773, 114

Proctor, D. D. 2006, ApJS, 165, 95

Reiprich, T. H., \& Böhringer, H. 2002, ApJ, 567, 716

Rettura, A., Martinez-Manso, J., Stern, D., et al. 2014, ApJ, 797, 109

Sarazin, C. L. 1988, X-ray emission from clusters of galaxies Schuster, M. T., Marengo, M., \& Patten, B. M. 2006, in Society of Photo-Optical Instrumentation Engineers (SPIE) Conference Series, Vol. 6270, Society of Photo-Optical Instrumentation Engineers (SPIE) Conference Series, 20

Simpson, C., \& Rawlings, S. 2002, MNRAS, 334, 511

Snyder, G. F., Brodwin, M., Mancone, C. M., et al. 2012, ApJ, 756, 114
Stanford, S. A., Gonzalez, A. H., Brodwin, M., et al. 2014, ApJS, 213, 25

Stanford, S. A., Eisenhardt, P. R., Brodwin, M., et al. 2005, ApJL, 634, L129

Stanford, S. A., Brodwin, M., Gonzalez, A. H., et al. 2012, ApJ, 753, 164

Sunyaev, R. A., \& Zeldovich, Y. B. 1970, Ap\&SS, 7, 3

Vanderlinde, K., Crawford, T. M., de Haan, T., et al. 2010, ApJ, 722, 1180

Venemans, B. P., Röttgering, H. J. A., Miley, G. K., et al. 2007, A\&A, 461, 823

Vikhlinin, A., Kravtsov, A. V., Burenin, R. A., et al. 2009, ApJ, 692, 1060

Werner, M. W., Roellig, T. L., Low, F. J., et al. 2004, ApJS, 154, 1

Wing, J. D., \& Blanton, E. L. 2011, AJ, 141, 88

—. 2013, ApJ, 767, 102

Wright, E. L. 2006, PASP, 118, 1711

Wylezalek, D., Galametz, A., Stern, D., et al. 2013, ApJ, 769,79

Wylezalek, D., Vernet, J., De Breuck, C., et al. 2014, ApJ, 786,17

Zeimann, G. R., Stanford, S. A., Brodwin, M., et al. 2012, ApJ, 756, 115

Zwicky, F., Herzog, E., Wild, P., Karpowicz, M., \& Kowal, C. T. 1961, Catalogue of galaxies and of clusters of galaxies, Vol. I 